\title{
Ethyl Pyruvate Prevents Methyglyoxal-Induced Retinal Vascular Injury in Rats
}

\author{
Junghyun Kim, Yun Mi Lee, Chan-Sik Kim, Eunjin Sohn, Kyuhyung Jo, \\ So Dam Shin, and Jin Sook Kim
}

Korean Medicine Based Herbal Drug Research Group, Herbal Medicine Research Division, Korea Institute of Oriental Medicine, 1672 Yuseongdaero, Yuseong-gu, Daejeon 305-811, Republic of Korea

Correspondence should be addressed to Jin Sook Kim; jskim@kiom.re.kr

Received 11 January 2013; Accepted 31 January 2013

Academic Editor: Xiang-an Li

Copyright (c) 2013 Junghyun Kim et al. This is an open access article distributed under the Creative Commons Attribution License, which permits unrestricted use, distribution, and reproduction in any medium, provided the original work is properly cited.

Pyruvate is an endogenous antioxidant substance. The aim of this study was to investigate the protective effects of ethyl pyruvate (EP) on retinal vascular injury in diabetic retinopathy. To investigate the protective effect of EP on vascular cell apoptosis and blood-retinal barrier (BRB) breakage, we have used intravitreally methylglyoxal-(MGO-) injected rat eyes. Apoptosis of the retinal vascular cell that was stimulated by the intravitreal injection of MGO was evidently attenuated by the EP treatment. EP exerts inhibitory effect on MGO-induced vascular cell apoptosis by blocking oxidative injury. In addition, EP treatment prevented MGOinduced BRB breakage and the degradation of occludin, an important tight junction protein. These observations suggest that EP acts through an antioxidant mechanism to protect against oxidative stress-induced apoptosis in retinal vessels.

\section{Introduction}

Diabetic retinopathy is one of the major complications of diabetic mellitus, and the main cause of acquired blindness in working-age adults. Retinal vascular injury, a hallmark of diabetic retinopathy, leads to the retinal pathological changes including thickening of the basement membrane, cellular capillary formation, retinal hemorrhage, endothelial proliferation, and angiogenesis, which ultimately leads to blindness [1-3]. Several studies demonstrated that retinal vascular cell apoptosis plays a crucial role in the development of diabetic retinopathy $[4,5]$.

Methylglyoxal (MGO) has been proposed for a causative factor of retinal vascular injury $[6,7]$. MGO, a glucosederived dicarbonyl intermediate, is found in high levels in blood or tissue of diabetic animals and patients $[8,9]$. MGO is more reactive than the parent sugars with respect to their abilities to cross-link with amino groups of various protein, forming stable end products called advanced glycation end products (AGEs) [10]. High levels of MGO affect cellular function by reacting with cellular proteins and nucleic acids [11]. There are reports suggesting that the cytotoxicity of MGO is due to its ability to induce apoptosis via oxidative stress [6, 12]. MGO can increase oxidative stress by modifying proteins associated with the formation of reactive oxygen species (ROS) and producing ROS during the glycation reaction [13-15].

Ethyl pyruvate (EP), a stable and lipophilic derivative of pyruvate, is considered to be an effective precursor of pyruvate [16]. The beneficial effects of EP as a potent inflammatory inhibitor and reactive oxygen species scavenger have been estimated in a variety of experimental animal models such as acute pancreatitis [17] and hepatic tumor [18]. EP has also shown renoprotective effect in streptozotocin-induced diabetic rats [19]. Despite the various effects of EP, knowledge of its mechanism of action and the effect on diabetic retinopathy is limited. The current study was conducted to investigate whether EP treatment was able to protect against MGOinduced retinal vascular injury and explore the underlying mechanisms in the intravitreally MGO-injected rat eyes.

\section{Materials and Methods}

2.1. Animals and Experimental Design. Thirty-two male SD rats (9 weeks old) were used in this study. Each rat was anesthetized with a 1:1 mixture xylazine hydrochloride $(4 \mathrm{mg} / \mathrm{kg})$ 
and ketamine hydrochloride $(10 \mathrm{mg} / \mathrm{kg})$. Sixteen rats were injected with a single dose of $6 \mathrm{mM}$ MGO in a volume $4 \mu \mathrm{L}$ into the vitreous of the right eye with a microinjector (Hamilton Co., NV, USA) under a dissecting microscope. For normal control, $3 \mu \mathrm{L}$ physiological saline was injected into the left eye. Sixteen rats were preinjected with $2 \mu \mathrm{L}$ EP $(300 \mathrm{mM})$ for $1 \mathrm{~h}$, followed by $2 \mu \mathrm{L}$ MGO $(12 \mathrm{mM})$ injection into the right eye. For negative control, $4 \mu \mathrm{L} \mathrm{EP}(150 \mathrm{mM})$ was injected into the left eye. Assuming the vitreous volume of an adult rat eye to be approximately $56 \mu \mathrm{L}$ [20], the final intravitreal concentration of MGO and EP were approximately $400 \mu \mathrm{M}$ and $10 \mathrm{mM}$, respectively. The dose of EP selected for this study was based upon a concentration of EP previously optimized in previous in vitro studies [16]. The needle was left in position for 30-60 s and then slowly withdrawn to minimize fluid loss from the eye. Rats were monitored regularly for infection associated with the injection site. Eyes with injection-damaged lenses or retinas were excluded from the study. At 2 days after the intravitreal injection, rats were anesthetized and killed. All experiments were approved by the Korea Institute of Oriental Medicine Institutional Animal Care and Use Committee.

2.2. Fluorescein-Dextran Microscopy. At necropsy, $1 \mathrm{~mL}$ of phosphate buffered saline (PBS) containing $50 \mathrm{mg}$ of fluorescein-dextran (Sigma, St. Louis, MO, USA) was injected into the left ventricle under deep anesthesia. The tracer was allowed to circulate for $10 \mathrm{~min}$ and eye was then enucleated and immediately fixed in $4 \%$ paraformaldehyde for 2 hours. The plasma was collected and assayed for fluorescence with a spectrofluorophotometer (Synergy ${ }^{\mathrm{TM}}$ HT, Bio-Tek, VT, USA) based on standard curves of FITC-dextran in normal plasma. The retinas were dissected, flat mounted onto a glass slide, and viewed by fluorescence microscopy (BX51, Olympus, Tokyo, Japan). Quantification of the fluorescence intensity was calculated by ImageJ software (NIH, MD, USA) and normalized to the plasma fluorescence intensity for each animal.

2.3. Preparation of Trypsin-Digested Vessels. The eyes were enucleated from the animals, and the retinas were isolated. After fixation $10 \%$ formalin for 2 days, the retinas were incubated in trypsin (3\% in sodium phosphate buffer containing $0.1 \mathrm{M}$ sodium fluoride to inhibit the DNase activity) for approximately $60 \mathrm{~min}$. The vessel structures were isolated from the retinal cells by gentle rinsing in distilled water. The vascular specimens were then mounted on slides.

2.4. Assessment of Apoptosis. Apoptosis was assessed using a TUNEL staining protocol according to the manufacturer's instructions (Promega, WI, USA). The number of TUNELpositive cells per unit area $\left(\mathrm{mm}^{2}\right)$ was then determined in and counted in a total of 5 fields. The numbers of apoptotic and total cells were counted.

2.5. Immunofluorescence Staining. The trypsin digests were immunofluorescently stained as previously described [18]. The slides were incubated with a mouse anti-8-hydroxyguanine (8-OHdG) antibody (Santa Cruz, CA, SUA) and a mouse antioccludin antibody (Invitrogen, CA, USA) for $1 \mathrm{~h}$. To detect 8-OHdG and occludin, the slides were incubated with a rodamine-conjugated goat anti-mouse antibody (Santa Cruz). The oxidation of guanine to form 8-OHdG acts as a marker of oxidative DNA damage [19]. The number of 8OHdG-positive cells was counted per five randomly selected $\mathrm{mm}^{2}$ of capillary area using ImageJ software (NIH).

2.6. Statistical Analysis. The results were evaluated statistically using one-way analysis of variance followed by Tukey's multiple comparison test using GraphPad Prism 4.0 (GraphPad Softwere, CA, USA).

\section{Results}

3.1. EP Prevented Apoptosis of Retinal Microvascular Cells. To characterize injury of retinal vascular pericyte and endothelial cell by MGO, TUNEL staining was performed using trypsin-digested retinal vessels. TUNEL analysis can detect cells in which DNA is fragmenting and is therefore widely used as a marker for apoptosis [21]. In the retinal trypsin digests of the saline-injected eyes, a TUNEL-positive nucleus was rarely detected (Figure 1(a)). In the MGO-injected eyes, many TUNEL-positive microvascular cells and fragmented nuclei were observed (Figure 1(b)). However, treatment of the MGO-injected eyes with EP prevented the increase in the positive cells that was seen in MGO-injected eyes (Figures $1(\mathrm{c})$ and $1(\mathrm{~d})$ ).

3.2. EP Inhibited Oxidative DNA Damage in Retinal Microvascular Cells. Representative patterns of the immunohistochemical localization of 8 -OHdG in the trypsin-digested retinal vessels are shown in Figure 2. The formation of 8$\mathrm{OHdG}$-adducted bases is an oxidative modification of DNA and is considered as a deteriorative consequence of oxidative stress [22]. 8-OHdG marker shows nuclear and/or perinuclear localization in retinal vascular pericytes and endothelial cells. Increased immunoreactivity of 8-OHdG was observed in the MGO-injected eyes. However, the treatment with EP suppressed the expression of 8 -OHdG compared to the MGO-injected eyes (Figure 2(d)).

3.3. EP Inhibited Blood-Retinal Barrier Breakage. A major clinical hallmark of diabetic retinopathy is increased capillary permeability culminating in an overt breakdown of the inner blood-retinal barrier (BRB) [21, 23]. In our previous study, MGO disrupts the tight junction protein, leading to breakage of the BRB [24]. EP was tested for its ability to inhibit $\mathrm{BRB}$ breakage in retinas. To evaluate increased vascular permeability in MGO-injected eyes, fluorescein angiography was performed using FTIC-dextran. Figure 3 shows representative fluorescence micrographs of FITC-dextran in control and MGO-injected eyes. The fluorescence intensity diffusely increased throughout the retinal parenchyma in the MGO-injected eyes but is limited to the vasculature in the control and EP-treated eyes. Figure 3(d) shows the change in the fluorescence intensity in the retinas after normalizing to plasma fluorescence. MGO increased retinal fluorescence by $75 \%(P<0.01)$. However, the treatment with EP significantly 


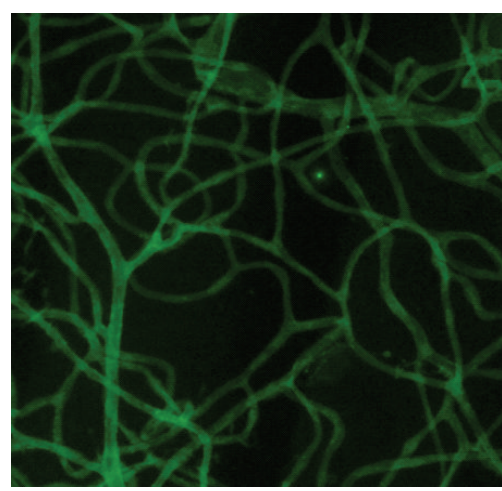

(a)

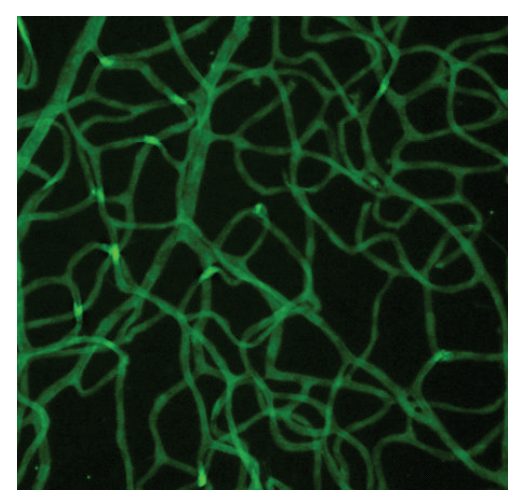

(b)

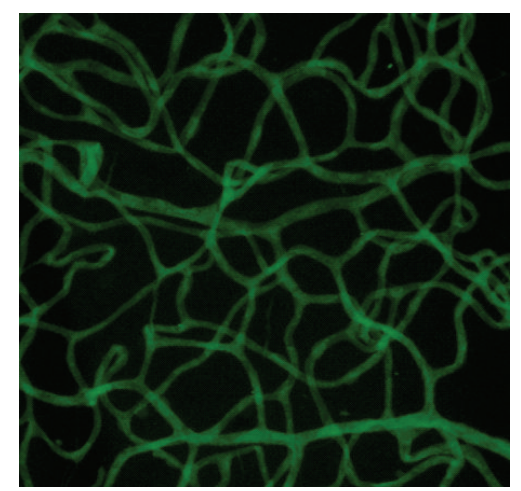

(c)

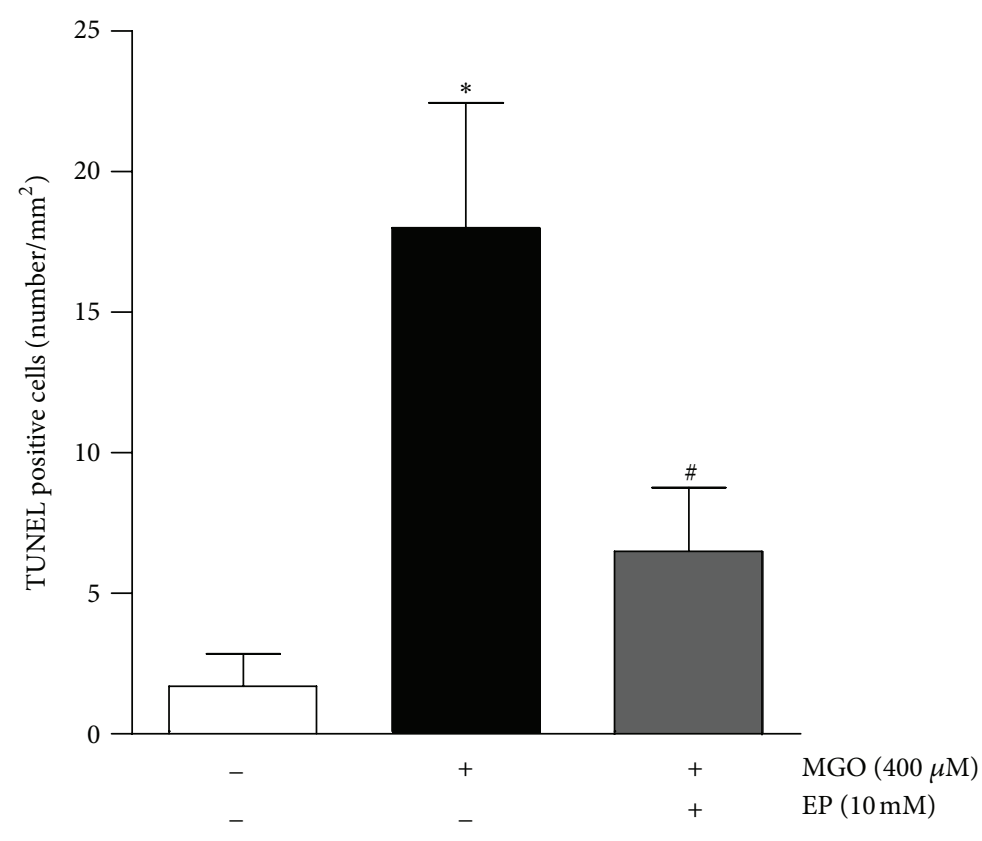

(d)

FIGURE 1: Retinal vascular cell apoptosis in intravitreally MGO-injected rat eyes. The trypsin-digested retinal vessels from a saline-injected eye (a), a MGO-injected eye (b), and an EP-treated eye (c) were stained with TUNEL (green). (d) Quantitative analysis of the TUNEL-positive cells. The values in the bar graphs represent the means \pm SE, $n=8 .{ }^{*} P<0.01$ versus the control group, ${ }^{\#} P<0.01$ versus the MGO-injected group.

decreased the fluorescence intensity in the MGO-injected eyes $(P<0.01)$.

3.4. EP Prevented Tight Junction Protein Loss. The loosening of the tight junctions increases retinal vascular permeability [25]. We investigated the expression of tight junction protein known as occludin. As shown in Figure 4, a marked decrease in occludin was detected in the MGO-injected eyes (white arrow). However, treatment of the MGO-injected eyes with EP prevented the loss of occludin in the MGO-injected eyes.

\section{Discussion}

Several studies demonstrated that retinal microvascular cell apoptosis and BRB breakage play a crucial role in the development of early diabetic retinopathy $[4,5]$. In the present study, in order to verify the therapeutic effects of EP in diabetic retinopathy, we investigated whether EP prevented this retinal vascular injury in MGO-injected eyes.

Pyruvate is the conjugate anion of pyruvic acid. Pyruvic acid is the final product of glycolysis and the initial substrate for the tricarboxylic acid cycle. The effects of pyruvate on 


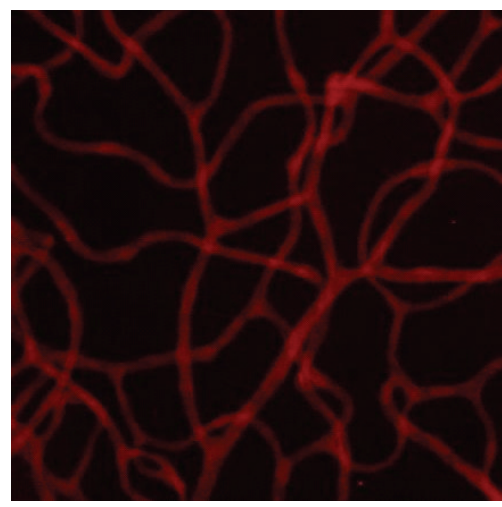

(a)

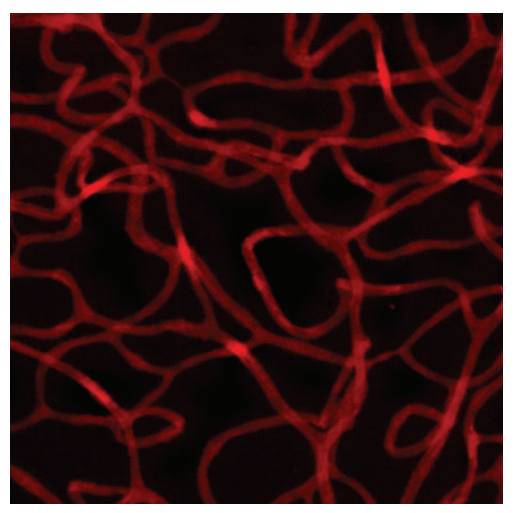

(b)

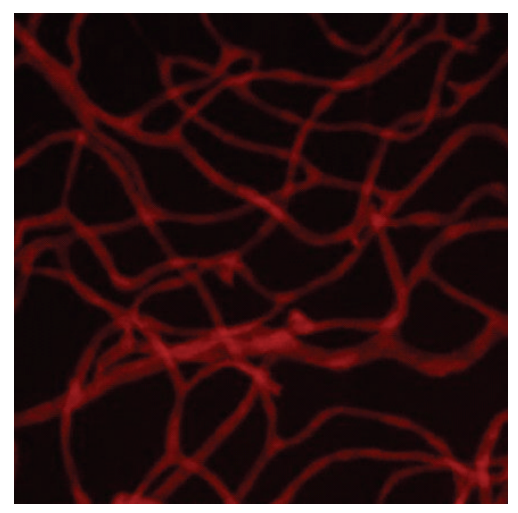

(c)

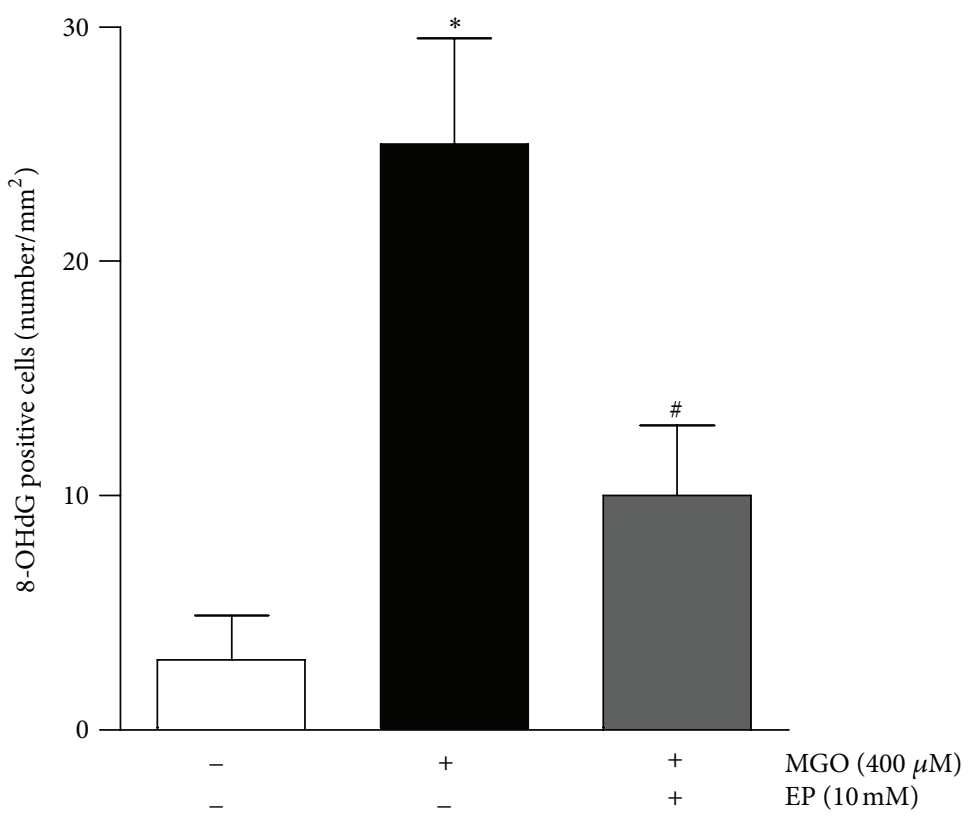

(d)

FIGURE 2: Oxidative DNA damage in retinal vessels derived from intravitreally MGO-injected rat eyes. The retinal tissues were stained with 8OHdG, which is a marker for oxidative DNA damage (red). Representative photomicrographs of the retinal vasculature from a saline-injected eye (a), a MGO-injected eye (b), and an EP-treated eye (c). (d) Quantitative analysis of the 8-OHdG-positive cells. The data are expressed as the means \pm SE. $(n=8) .{ }^{*} P<0.01$ versus the control group, ${ }^{*} P<0.01$ versus the MGO-injected group.

scavenging reactive oxygen radicals have been well known. It became of clinical interest, since reactive oxygen species have been implicated in various injury models. However, the pharmacological application of pyruvate is limited because it is not stable in solution and spontaneously forms potentially toxic metabolites. However, ethyl pyruvate, a derivate of pyruvic acid, is stable in solution and nontoxic [26]. Ethyl pyruvate has demonstrated protective effects in pancreatitis [27], hemorrhagic shock [28] and $\mathrm{H}_{2} \mathrm{O}_{2}$-induced renal injury [29] as well as diabetic nephropathy [19] and liver injury [30]. In addition, there is evidence that ethyl pyruvate can ameliorate vascular injury by inhibiting ROS production and downregulating inflammatory mediators [31, 32].
Several studies have been reported that the cell death induced by MGO appeared to be due to apoptosis in a variety of cell types $[12,33,34]$. Recently, it was reported that MGO induced the apoptosis of bovine retinal pericytes [6]. In our previous study, MGO is a cause of apoptosis of cultured rat retinal pericytes [35]. MGO has been reported to induce apoptotic cell death via various mechanisms, such as ROS generation [36], p38 MAPK activation [34], JNK pathway [12], and alteration of PDGFR signaling [37]. Recently, it was reported that MGO induced the apoptosis of bovine retinal pericytes by oxidative stress [6]. The treatment with the ROS scavenger, $\mathrm{N}$-acetyl cysteine, attenuated MGO-induced apoptotic cell death in rat retinal pericyte [35]. Moreover, in 


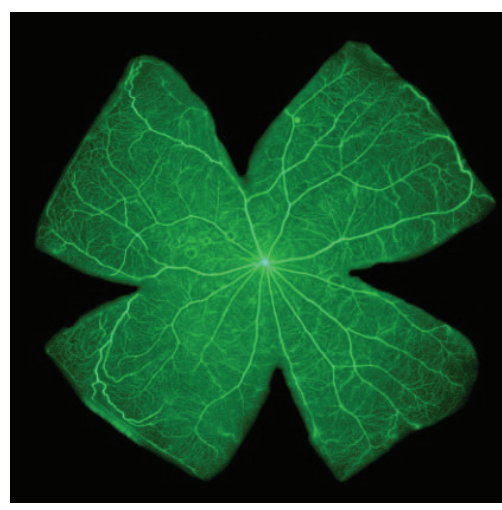

(a)

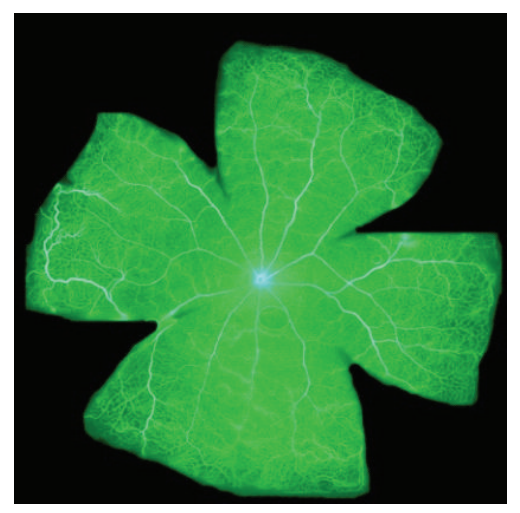

(b)

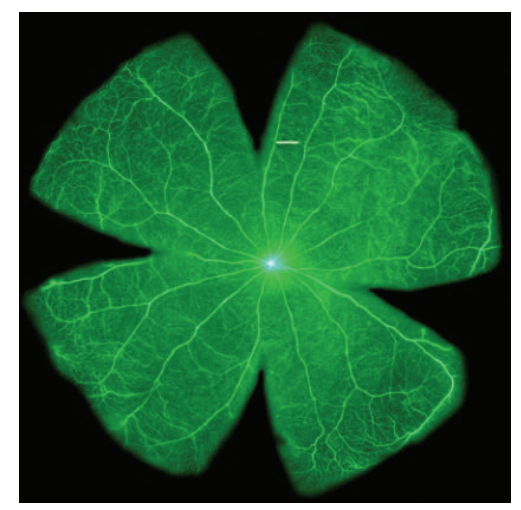

(c)

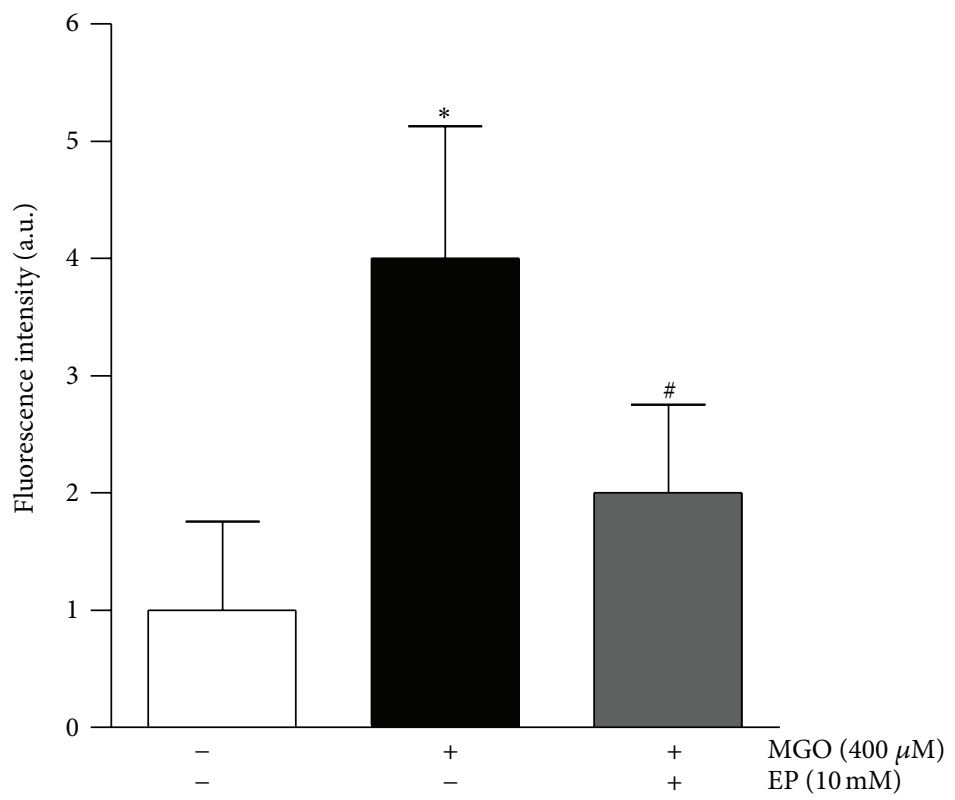

(d)

FIGURE 3: Blood-retinal barrier breakdown. Retinal permeability was determined by the FITC-dextran technique. Representative photomicrographs of the retina from a saline-injected eye (a), a MGO-injected eye (b), and an EP-treated eye (c). Control retina showed no leakage of the tracer into the retina as evidenced by the clear delineation of retinal capillaries. MGO-injected eye demonstrated a widespread breakdown of their BRB with tracer leakage into the neural retina and a loss of delineation of the retinal capillaries. However, the treatment of EP significantly decreased retinal vascular permeability. (d) Quantitative analysis of the immunofluorescence intensity for FITC-dextran. The data are expressed as the means \pm SE. $(n=8) .{ }^{*} P<0.01$ versus the control group, ${ }^{\#} P<0.01$ versus the MGO-injected group.

diabetic rats, a mixture of several antioxidants [4] or trolox [38] was able to prevent pericyte loss in retina. To elucidate the mechanism involved in the antiapoptotic effect of EP in rat retinal microvascular cells, we focused on MGO-induced oxidative stress. In the present study, EP inhibited the increase in oxidative DNA damage induced by MGO. When DNA is damaged, cells initiate a cell cycle delay or induction of apoptosis [39]. Diabetes-induced oxidative stress has been well documented in patients and animals [40-42]. Formation of AGEs has been shown to be a source of oxidative stress in retinal endothelial cells via the interaction with AGE receptor [43]. Our results indicate that EP is capable of preventing
MGO-induced oxidative stress by virtue of its ability to act as an ROS scavenger.

The destruction of retinal vascular pericytes and endothelial cells results in the formation of acellular capillaries, which are associated with areas of nonperfusion. The increasing of acellular capillaries then leads to the development of retinal ischemia and retinal neovascularization. Therefore, inhibition of apoptosis by EP could be expected to inhibit the development of retinal ischemia and neovascularization. Furthermore, the fluorescein angiography showed that EP markedly inhibited the fluorescein leakage, which suggests that EP might prevent the breakdown of BRB. MGO has 


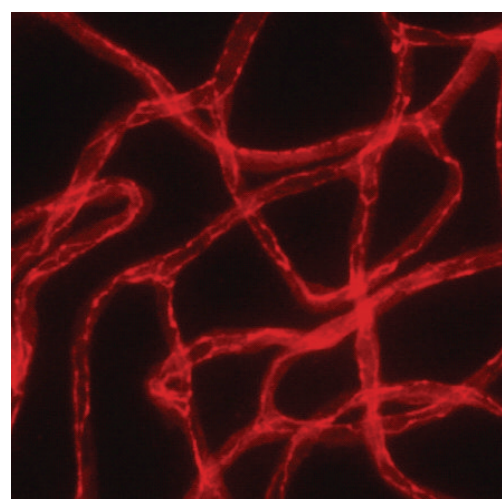

(a)

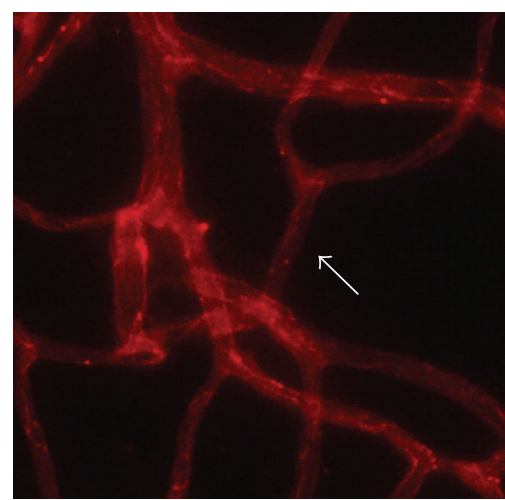

(b)

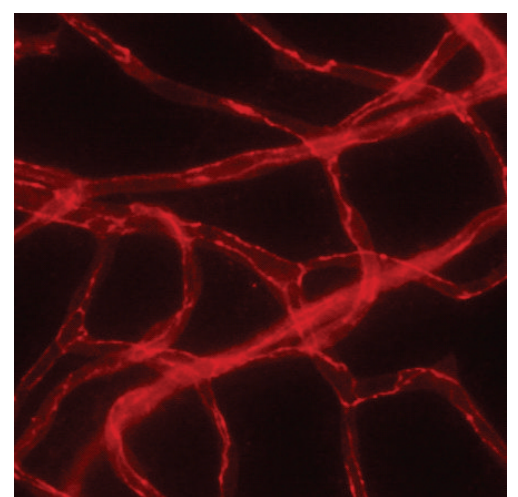

(c)

FIGURE 4: MGO-induced occludin loss. Representative retinal vessels from a saline-injected eye (a), a MGO-injected eye (b), and an EPtreated eye (c) were stained with antioccludin antibody. Occludin expression was evident at the interfaces between adjacent endothelial cells in the control eyes, while it was mostly eliminated from the microvessels in the MGO-injected eyes (arrow).

been shown to induce ROS generation in the endothelium [44]. Oxidative stress is known for its deleterious effects, which can cause enhanced permeability of the BRB $[45,46]$. The retinal endothelial monolayer exhibits high transendothelial resistance, a property that is attributed to intercellular tight junctions between apposed endothelial cells [47]. Among the several tight-junctional proteins, occludin is pivotal in the regulation of endothelial monolayer electrical resistance, paracellular solute permeability, and normal barrier function [48]. Although occludin by itself cannot form a functionally tight barrier, it likely plays an important role in the organization and stabilization of the tight junction. MGO-induced tight-junctional disruption could be a mechanism in barrier impairment associated with vascular pathology, such as in diabetes $[9,49]$. Therefore, MGO-mediated disruption of occludin is expected to increase endothelial permeability, and the attenuation of MGO stress would preserve barrier integrity. Indeed, our result showed that MGO-induced occludin degradation was attenuated by the treatment of EP. The results suggest that $\mathrm{EP}$ also has potential to prevent $\mathrm{BRB}$ dysfunction induced by MGO.

In conclusion, our study shows that $\mathrm{EP}$ inhibits the retinal vascular cell apoptosis and BRB breakage induced by MGO in rats. In addition, oxidative stress induced by MGO was significantly inhibited by treatment with EP. The attenuation of MGO stress by EP preserved the tight junction protein occludin integrity. These observations suggest that EP acts through an antioxidant mechanism to protect against oxidative stress-induced retinal vascular injury. Taken together, these results indicate that treatment with EP could be a valuable therapeutic approach in the treatment or prevention of diabetic vascular injury.

\section{Conflict of Interests}

The authors declare no conflict of interests.

\section{Acknowledgment}

This research was supported by a Grant (K12040) from the Korea Institute of Oriental Medicine (KIOM).

\section{References}

[1] B. H. Chen, D. Y. Jiang, and L. S. Tang, "Advanced glycation endproducts induce apoptosis involving the signaling pathways of oxidative stress in bovine retinal pericytes," Life Sciences, vol. 79, no. 11, pp. 1040-1048, 2006.

[2] H. P. Hammes, J. Lin, O. Renner et al., "Pericytes and the pathogenesis of diabetic retinopathy," Diabetes, vol. 51, no. 10, pp. 3107-3112, 2002.

[3] S. Roy, T. Sato, G. Paryani, and R. Kao, "Downregulation of fibronectin overexpression reduces basement membrane thickening and vascular lesions in retinas of galactose-fed rats," Diabetes, vol. 52, no. 5, pp. 1229-1234, 2003.

[4] S. Yatoh, M. Mizutani, T. Yokoo et al., "Antioxidants and an inhibitor of advanced glycation ameliorate death of retinal microvascular cells in diabetic retinopathy," Diabetes/Metabolism Research and Reviews, vol. 22, no. 1, pp. 38-45, 2006.

[5] H. P. Hammes, "Pericytes and the pathogenesis of diabetic retinopathy," Hormone and Metabolic Research, vol. 37, supplement 1, pp. S39-S43, 2005.

[6] J. Kim, J. Son, J. Lee, Y. Oh, and S. Shinn, "Methylglyoxal induces apoptosis mediated by reactive oxygen species in bovine retinal pericytes," Journal of Korean Medical Science, vol. 19, no. 1, pp. 95-100, 2004.

[7] A. G. Miller, D. G. Smith, M. Bhat, and R. H. Nagaraj, "Glyoxalase I is critical for human retinal capillary pericyte survival under hyperglycemic conditions," The Journal of Biological Chemistry, vol. 281, no. 17, pp. 11864-11871, 2006.

[8] B. K. Kilhovd, I. Giardino, P. A. Torjesen et al., "Increased serum levels of the specific AGE-compound methylglyoxal-derived hydroimidazolone in patients with type 2 diabetes," Metabolism, vol. 52, no. 2, pp. 163-167, 2003. 
[9] A. Lapolla, R. Flamini, A. Dalla Vedova et al., "Glyoxal and methylglyoxal levels in diabetic patients: quantitative determination by a new GC/MS method," Clinical Chemistry and Laboratory Medicine, vol. 41, no. 9, pp. 1166-1173, 2003.

[10] M. Bourajjaj, C. D. Stehouwer, V. W. van Hinsbergh, and C. G. Schalkwijk, "Role of methylglyoxal adducts in the development of vascular complications in diabetes mellitus," Biochemical Society Transactions, vol. 31, no. 6, pp. 1400-1402, 2003.

[11] J. Zeng and M. J. Davies, "Evidence for the formation of adducts and S-(carboxymethyl)cysteine on reaction of $\alpha$-dicarbonyl compounds with thiol groups on amino acids, peptides, and proteins," Chemical Research in Toxicology, vol. 18, no. 8, pp. 1232-1241, 2005.

[12] W. H. Chan, H. J. Wu, and N. H. Shiao, "Apoptotic signaling in methylglyoxal-treated human osteoblasts involves oxidative stress, c-Jun N-terminal kinase, caspase-3, and p21-activated kinase 2," Journal of Cellular Biochemistry, vol. 100, no. 4, pp. 1056-1069, 2007.

[13] K. M. Beard, N. Shangari, B. Wu, and P. J. O’Brien, "Metabolism, not autoxidation, plays a role in $\alpha$-oxoaldehyde- and reducing sugar-induced erythrocyte GSH depletion: relevance for diabetes mellitus," Molecular and Cellular Biochemistry, vol. 252, no. 1-2, pp. 331-338, 2003.

[14] H. S. Yim, S. O. Kang, Y. C. Hah, P. B. Chock, and M. B. Yim, "Free radicals generated during the glycation reaction of amino acids by methylglyoxal. A model study of protein-cross-linked free radicals," The Journal of Biological Chemistry, vol. 270, no. 47, pp. 28228-28233, 1995.

[15] D. L. V. Jagt, L. A. Hunsaker, T. J. V. Jagt et al., "Inactivation of glutathione reductase by 4-hydroxynonenal and other endogenous aldehydes," Biochemical Pharmacology, vol. 53, no. 8, pp. 1133-1140, 1997.

[16] K. K. Kao and M. P. Fink, "The biochemical basis for the antiinflammatory and cytoprotective actions of ethyl pyruvate and related compounds," Biochemical Pharmacology, vol. 80, no. 2, pp. 151-159, 2010.

[17] Z. Y. Yang, Y. Ling, T. Yin et al., "Delayed ethyl pyruvate therapy attenuates experimental severe acute pancreatitis via reduced serum high mobility group box 1 levels in rats," World Journal of Gastroenterology, vol. 14, no. 28, pp. 4546-4550, 2008.

[18] X. Liang, A. R. D. V. Chavez, N. E. Schapiro et al., "Ethyl pyruvate administration inhibits hepatic tumor growth," Journal of Leukocyte Biology, vol. 86, no. 3, pp. 599-607, 2009.

[19] K. D. Ju, E. K. Shin, E. J. Cho et al., "Ethyl pyruvate ameliorates albuminuria and glomerular injury in the animal model of diabetic nephropathy," American Journal of Physiology, vol. 302, no. 5, pp. F606-F613, 2012.

[20] B. A. Berkowitz, R. A. Lukaszew, C. M. Mullins, and J. S. Penn, "Impaired hyaloidal circulation function and uncoordinated ocular growth patterns in experimental retinopathy of prematurity," Investigative Ophthalmology and Visual Science, vol. 39, no. 2, pp. 391-396, 1998.

[21] A. Patz, "I. Studies on retinal neovascularization. Friedenwald lecture," Investigative Ophthalmology and Visual Science, vol. 19, no. 10, pp. 1133-1138, 1980.

[22] C. Li, K. Wu, G. Chang-Chien, and C. Chou, "Analysis of oxidative DNA damage 8-hydroxy-2'-deoxyguanosine as a biomarker of exposures to persistent pollutants for marine mammals," Environmental Science and Technology, vol. 39, no. 8, pp. 2455-2460, 2005.

[23] B. Sander, M. Larsen, C. Engler, H. Lund-Andersen, and H. H. Parving, "Early changes in diabetic retinopathy: capillary loss and blood-retina barrier permeability in relation to metabolic control," Acta Ophthalmologica, vol. 72, no. 5, pp. 553-559, 1994.

[24] J. Kim, C. Kim, Y. M. Lee, K. Jo, S. D. Shin, and J. S. Kim, "Methylglyoxal induces hyperpermeability of the blood-retinal barrier via the loss of tight junction proteins and the activation of matrix metalloproteinases," Graefe's Archive for Clinical and Experimental Ophthalmology, vol. 250, no. 5, pp. 691-697, 2012.

[25] S. J. Giebel, G. Menicucci, P. G. McGuire, and A. Das, "Matrix metalloproteinases in early diabetic retinopathy and their role in alternation of the blood-retinal barrier," Laboratory Investigation, vol. 85, no. 5, pp. 597-607, 2005.

[26] M. P. Fink, "Ethyl pyruvate: a novel treatment for sepsis and shock," Minerva Anestesiologica, vol. 70, no. 5, pp. 365-371, 2004.

[27] R. Yang, T. Uchiyama, S. M. Alber et al., "Ethyl pyruvate ameliorates distant organ injury in a murine model of acute necrotizing pancreatitis," Critical Care Medicine, vol. 32, no. 7, pp. 14531459, 2004.

[28] R. Venkataraman, J. A. Kellum, M. Song, and M. P. Fink, "Resuscitation with Ringer's ethyl pyruvate solution prolongs survival and modulates plasma cytokine and nitrite/nitrate concentrations in a rat model of lipopolysaccharide-induced shock," Shock, vol. 18, no. 6, pp. 507-512, 2002.

[29] A. K. Salahudeen, E. C. Clark, and K. A. Nath, "Hydrogen peroxide-induced renal injury. A protective role for pyruvate in vitro and in vivo," The Journal of Clinical Investigation, vol. 88, no. 6, pp. 1886-1893, 1991.

[30] H. Akkoc, I. Kelle, S. Tunik et al., "Protective effect of ethyl pyruvate on liver injury in streptozotocin-induced diabetic rats," Acta Gastro-Enterologica Belgica, vol. 75, no. 3, pp. 336-341, 2012.

[31] C. M. Corl, H. R. Robinson, G. A. Contreras, S. J. Holcombe, V. L. Cook, and L. M. Sordillo, "Ethyl pyruvate diminishes the endotoxin-induced inflammatory response of bovine mammary endothelial cells," Journal of Dairy Science, vol. 93, no. 11, pp. 5188-5199, 2010.

[32] M. M. Rabadi, T. Ghaly, M. S. Goligorksy, and B. B. Ratliff, "HMGB1 in renal ischemic injury," American Journal of Physiology, vol. 303, no. 6, pp. 873-885, 2012.

[33] S. M. Huang, H. C. Chuang, C. H. Wu, and G. C. Yen, "Cytoprotective effects of phenolic acids on methylglyoxal-induced apoptosis in Neuro-2A cells," Molecular Nutrition and Food Research, vol. 52, no. 8, pp. 940-949, 2008.

[34] B. Liu, S. Miyata, Y. Hirota et al., "Methylglyoxal induces apoptosis through activation of $\mathrm{p} 38$ mitogen-activated protein kinase in rat mesangial cells," Kidney International, vol. 63, no. 3, pp. 947-957, 2003.

[35] O. S. Kim, J. Kim, C. Kim, N. H. Kim, and J. S. Kim, "KIOM79 prevents methyglyoxal-induced retinal pericyte apoptosis in vitro and in vivo," Journal of Ethnopharmacology, vol. 129, no. 3, pp. 285-292, 2010.

[36] J. Du, H. Suzuki, F. Nagase et al., "Superoxide-mediated early oxidation and activation of ASK1 are important for initiating methylglyoxal-induced apoptosis process," Free Radical Biology and Medicine, vol. 31, no. 4, pp. 469-478, 2001.

[37] A. V. Cantero, M. Portero-Otín, V. Ayala et al., "Methylglyoxal induces advanced glycation end product (AGEs) formation and dysfunction of PDGF receptor- $\beta$ : implications for diabetic atherosclerosis," The FASEB Journal, vol. 21, no. 12, pp. 30963106, 2007.

[38] N. H. Ansari, W. Zhang, E. Fulep, and A. Mansour, "Prevention of pericyte loss by trolox in diabetic rat retina," Journal of 
Toxicology and Environmental Health A, vol. 54, no. 6, pp. 467475, 1998.

[39] O. Zgheib, Y. Huyen, R. A. DiTullio Jr. et al., "ATM signaling and 53BP1," Radiotherapy and Oncology, vol. 76, no. 2, pp. 119-122, 2005.

[40] A. Ceriello, F. Mercuri, L. Quagliaro et al., "Detection of nitrotyrosine in the diabetic plasma: evidence of oxidative stress," Diabetologia, vol. 44, no. 7, pp. 834-838, 2001.

[41] L. Fathallah and I. G. Obrosov, "Increased retinal lipid peroxidation in early diabetes is not associated with ascorbate depletion or changes in ascorbate redox state," Experimental Eye Research, vol. 72, no. 6, pp. 719-723, 2001.

[42] D. Armstrong and F. Al-Awadi, "Lipid peroxidation and retinopathy in streptozotocin-induced diabetes," Free Radical Biology and Medicine, vol. 11, no. 4, pp. 433-436, 1991.

[43] A. W. Stitt, "The role of advanced glycation in the pathogenesis of diabetic retinopathy," Experimental and Molecular Pathology, vol. 75, no. 1, pp. 95-108, 2003.

[44] N. Miyazawa, M. Abe, T. Souma et al., "Methylglyoxal augments intracellular oxidative stress in human aortic endothelial cells," Free Radical Research, vol. 44, no. 1, pp. 101-107, 2010.

[45] R. Chibber, B. M. Ben-Mahmud, S. Chibber, and E. M. Kohner, "Leukocytes in diabetic retinopathy," Current Diabetes Reviews, vol. 3, no. 1, pp. 3-14, 2007.

[46] A. B. El-Remessy, M. Al-Shabrawey, Y. Khalifa, N. Tsai, R. B. Caldwell, and G. I. Liou, "Neuroprotective and blood-retinal barrier-preserving effects of cannabidiol in experimental diabetes," American Journal of Pathology, vol. 168, no. 1, pp. 235244, 2006.

[47] P. M. Watson, J. M. Anderson, C. M. Vanltallie, and S. R. Doctrow, "The tight-junction-specific protein $\mathrm{ZO}-1$ is a component of the human and rat blood-brain barriers," Neuroscience Letters, vol. 129, no. 1, pp. 6-10, 1991.

[48] B. T. Hawkins and T. P. Davis, "The blood-brain barrier/neurovascular unit in health and disease," Pharmacological Reviews, vol. 57, no. 2, pp. 173-185, 2005.

[49] P. J. Beisswenger, S. K. Howell, A. D. Touchette, S. Lal, and B. S. Szwergold, "Metformin reduces systemic methylglyoxal levels in type 2 diabetes," Diabetes, vol. 48, no. 1, pp. 198-202, 1999. 


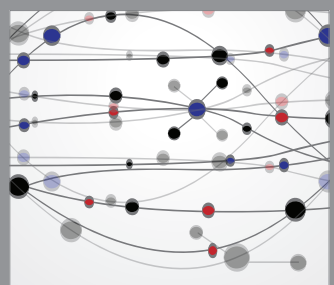

The Scientific World Journal
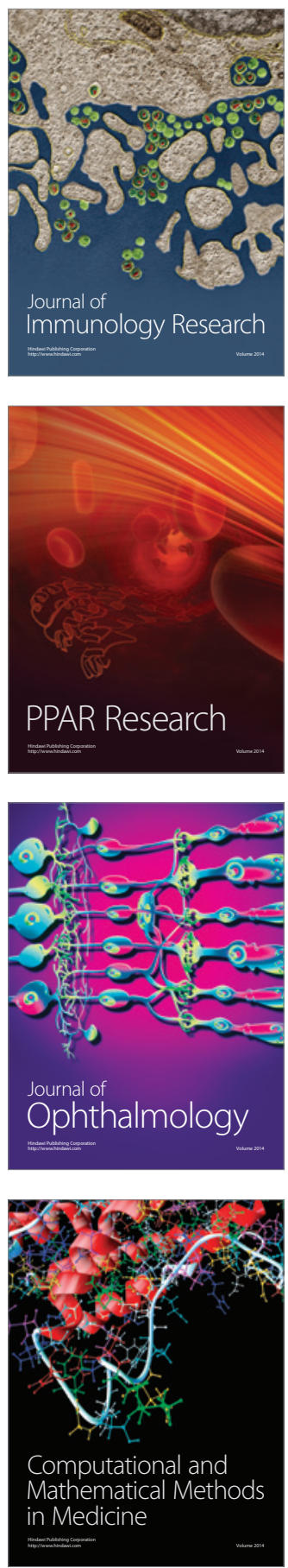

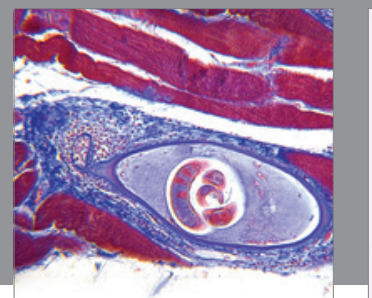

Gastroenterology

Research and Practice
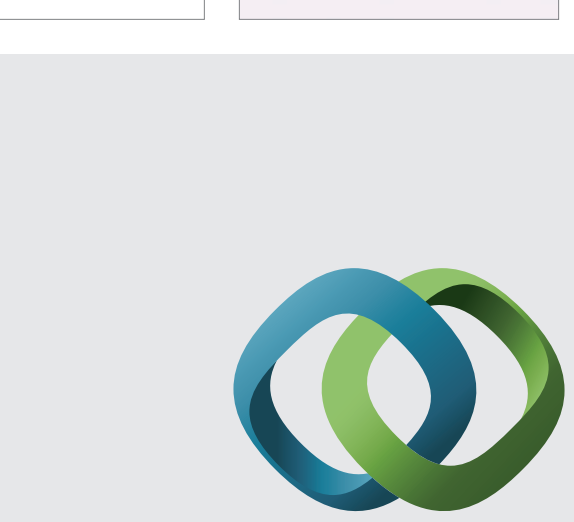

\section{Hindawi}

Submit your manuscripts at

http://www.hindawi.com
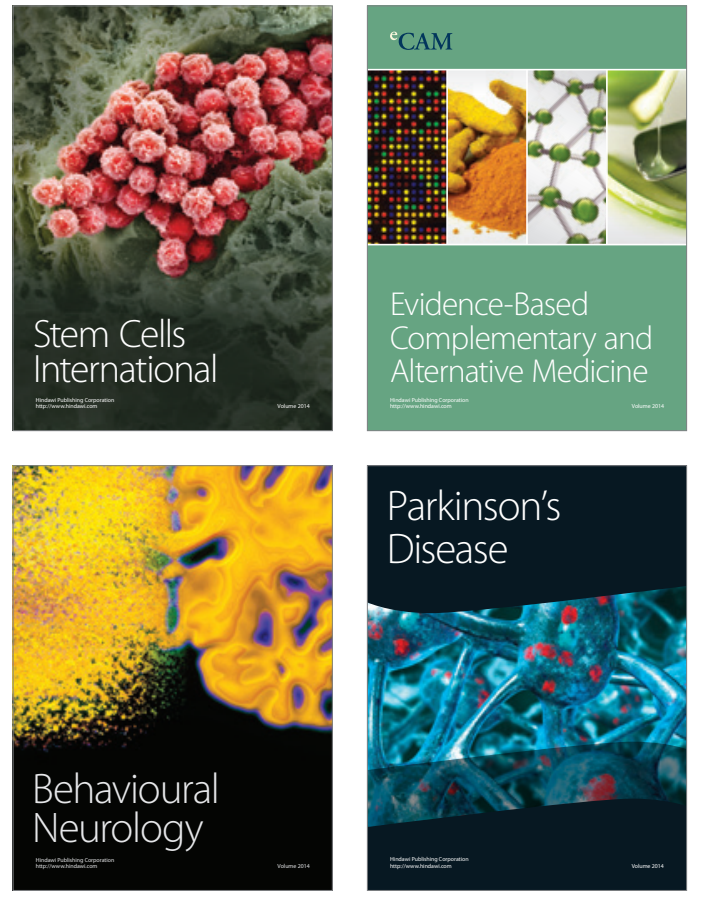
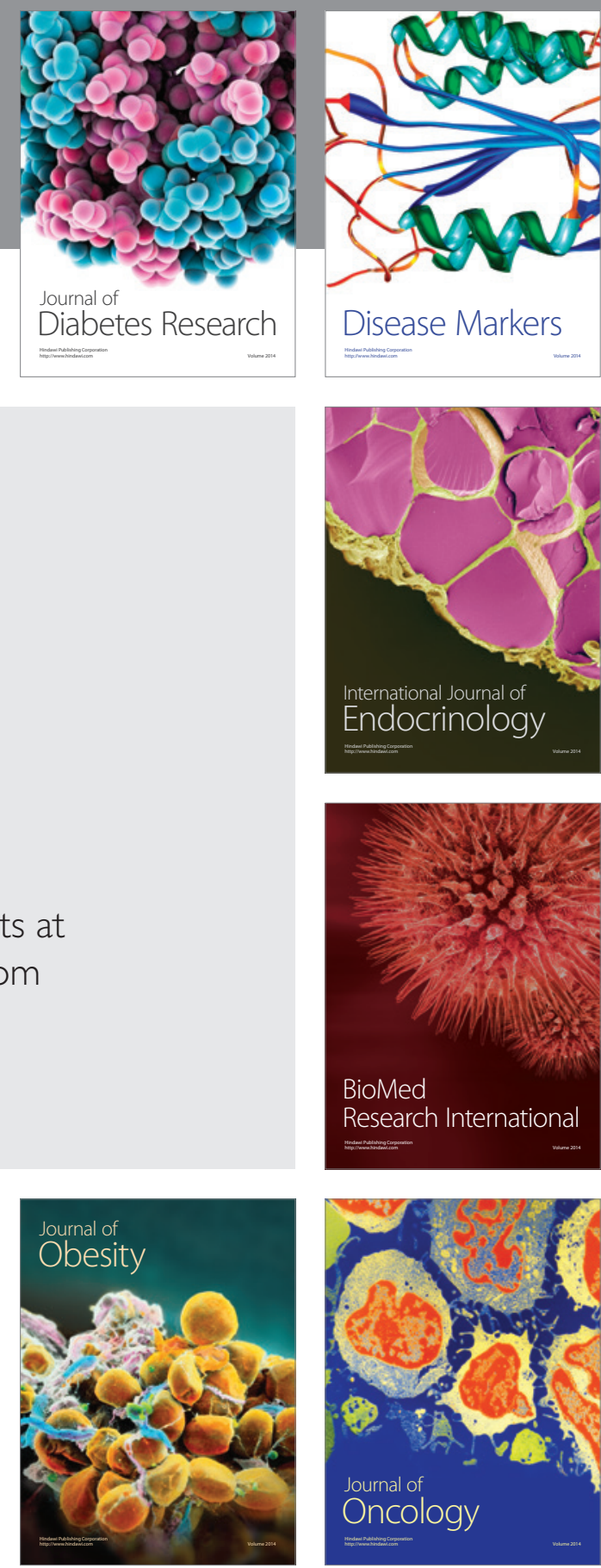

Disease Markers
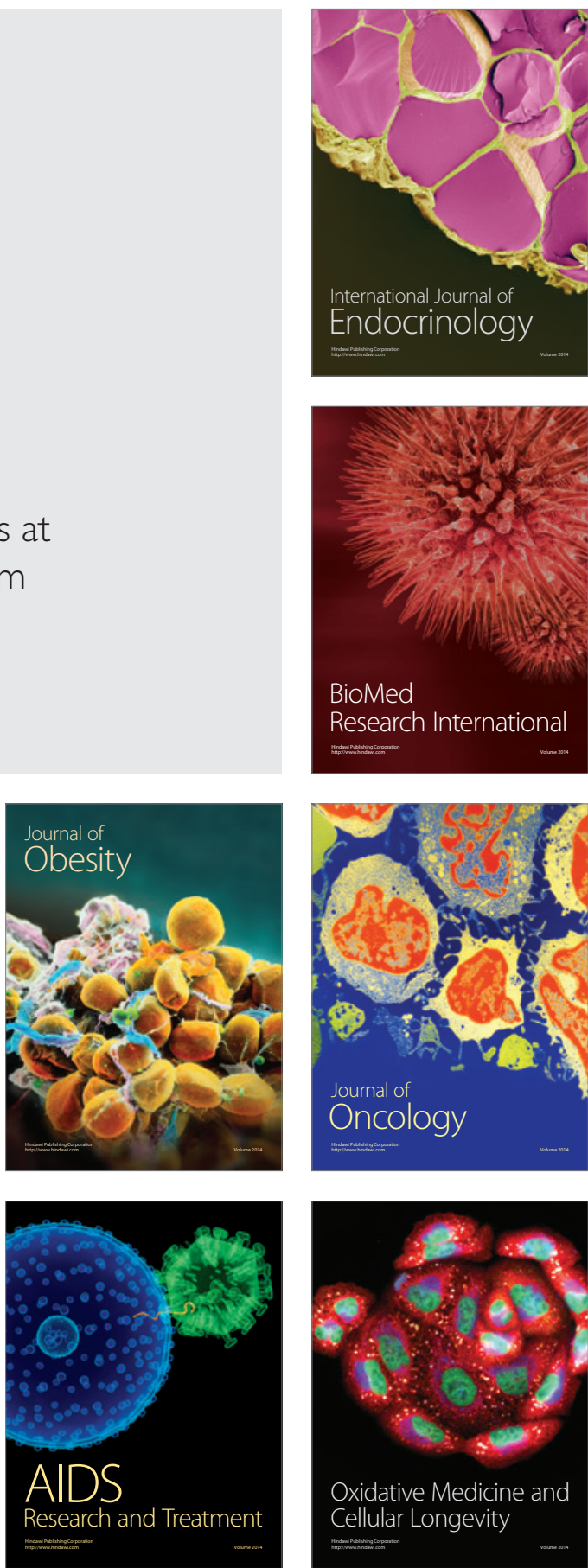